\title{
Surface brightness profile of the Milky Way's nuclear star cluster (Corrigendum)
}

\author{
R. Schödel ${ }^{1}$, A. Feldmeier ${ }^{2}$, D. Kunneriath ${ }^{3}$, S. Stolovy ${ }^{4}$, N. Neumayer ${ }^{2}$, P. Amaro-Seoane ${ }^{5}$, and S. Nishiyama ${ }^{6}$ \\ ${ }^{1}$ Instituto de Astrofísica de Andalucía (CSIC), Glorieta de la Astronomía s/n, 18008 Granada, Spain \\ e-mail: rainer@iaa.es \\ 2 European Southern Observatory, Karl-Schwarzschild-Strasse 2, 85748 Garching bei München, Germany \\ 3 Astronomical Institute, Academy of Sciences, Boční II 1401, 14100 Prague, Czech Republic \\ 4 El Camino College, 16007 Crenshaw Blvd., Torrance, CA 90506, USA \\ 5 Max-Planck Institut für Gravitationsphysik (Albert-Einstein-Institut), Am Mühlenberg 1, 14476 Potsdam, Germany \\ ${ }^{6}$ National Astronomical Observatory of Japan, Mitaka, 181-8588 Tokyo, Japan
}

A\&A 566, A47 (2014), DOI: 10.1051/0004-6361/201423481

Key words. dust, extinction - Galaxy: center - Galaxy: nucleus - Galaxy: structure - galaxies: nuclei - errata, addenda

The first column of Table 4 for the multi-Gaussian expansion (MGE) fits contains an error. The maximum intensity of the Gaussian components $I$ is in $10^{5} L_{\odot, 4.5 \mu \mathrm{m}} / \mathrm{pc}^{2}$ and not in

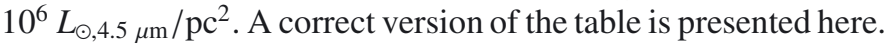

Acknowledgements. We thank Troy Raen for bringing this to our attention.
Table 4. MGE fit parameters for the Spitzer/IRAC PAH- and extinctioncorrected image.

\begin{tabular}{ccc}
\hline \hline $\begin{array}{c}I \\
\left(10^{5} L_{\left.\odot, 4.5 \mu \mathrm{m} / \mathrm{pc}^{2}\right)}\right.\end{array}$ & $\begin{array}{c}\sigma \\
(\operatorname{arcsec})\end{array}$ & $q$ \\
\hline 25.13 & 14.1 & 0.90 \\
2.63 & 52.8 & 1.00 \\
1.35 & 56.1 & 0.35 \\
2.33 & 101.6 & 0.38 \\
0.71 & 150.5 & 1.00 \\
0.15 & 481.3 & 0.09 \\
0.44 & 581.3 & 0.42 \\
0.17 & 2656.5 & 1.00 \\
0.31 & 2656.5 & 0.20 \\
\hline
\end{tabular}

